\title{
Modelo de previsão de demanda de energia elétrica para o campus sede da Universidade de Caxias do Sul empregando máquina de vetores de suporte
}

\author{
Vinícius Bortoluzzi*, André Bernardes Michel* \\ * Centro de Ciências Exatas, da Natureza e de Tecnologia, \\ Universidade de Caxias do Sul (UCS), Caxias do Sul, RS, Brasil \\ (e-mails:vbortoluzzi@ucs.br, abmichel@ucs.br)
}

\begin{abstract}
The forecast of demand for electric energy is often discussed in the literature, whether applied to a individual consumer or to a interconnected systems. Models are constructed based on historical data with the objective to describe the behavior of demand over a predetermined time horizon. In this work, the supervised machine learning method known as support vector machine is applied to develop a forecast model of demand for the campus of the Universidade de Caxias do Sul considering the medium term. The selection of the historical data that integrate the model is performed using the additive selection. For the determination of the parameters for the construction of the final model, the grid search method and genetic algorithm are used. The best model of this work presented a mean absolute percentage error of $5.72 \%, 4.26 \%$ inferior to the best model found in the literature.

Resumo: A previsão de demanda de energia elétrica é bastante discutida na literatura, seja aplicada à consumidores individuais ou à sistemas interligados. A partir de conjuntos de dados históricos são construídos modelos que tentam descrever o comportamento da demanda em um horizonte de tempo predeterminado. Neste trabalho, aplica-se o método de aprendizagem de máquina supervisionado conhecido como máquina de vetores de suporte, voltada à regressão, para desenvolver um modelo de previsão de demanda para o campus sede da Universidade de Caxias do Sul a médio prazo. A seleção dos dados históricos que integram o modelo é realizada por meio de seleção aditiva e são empregados, na determinação dos parâmetros para a construção do modelo final, os métodos busca em grade e algoritmo genético. $\mathrm{O}$ melhor modelo deste trabalho, cujo erro percentual absoluto médio foi de 5,72\%, distanciou-se em 4,26\% do melhor modelo encontrado na literatura.
\end{abstract}

Keywords: Machine Learning; Support Vector Machine; Forecast Model; Medium Term; Grid Search; Genetic Algorithm.

Palavras-chaves: Aprendizagem Máquina; Vetores Suporte; Previsão Demanda; Médio Prazo; Busca Grade; Algoritmo Genético.

\section{INTRODUÇÃO}

Métodos de previsão, independentemente dos problemas aos quais são aplicados, estão relacionados diretamente aos dados disponíveis. A escolha de um método também está relacionada, amplamente, às seguintes características almejadas: precisão, horizonte de previsão (curto, médio ou longo prazo), resiliência frente a novos cenários e recursos computacionais disponíveis à implementação (Ferreira, 2006).

No caso da previsão de demanda, objeto foco deste trabalho, a escolha de um horizonte de previsão é baseada no objetivo almejado. De um ponto de vista geral, enquanto previsões de curto prazo são empregadas na programação de despachos e na operação e coordenação de sistemas elétricos, previsões de médio e longo prazo visam fornecer informações que podem colaborar, tanto no planejamento de investimentos em expansão quanto na programação de aquisição de combustíveis (Al-Alawi and Islam, 1996).

Previsões cujos horizontes são o médio e o longo prazo envolvem diversos fatores exógenos, acarretando em uma complexidade maior do que a associada a uma previsão de curto prazo. Além de dados referentes às condições climáticas, é explícita a relação da demanda com indicadores econômicos, hábitos de consumo e fatores casuais, como feriados e eventos nacionais (Khuntia et al., 2016) e (AlAlawi and Islam, 1996).

No rol das variáveis climáticas figuram a temperatura, umidade, velocidade do vento, níveis de precipitação, nível de radiação, dentre outras. Pode-se citar como variáveis econômicas influentes a evolução do PIB (Produto Interno Bruto) e da população, por exemplo. A escolha das variáveis a serem consideradas na construção do modelo de 
previsão dependerá, fundamentalmente, das suas influências no horizonte almejado (Al-Alawi and Islam, 1996).

Em algumas abordagens, os dados de entrada podem ser submetidos a um pré-processamento. O intuito dessa operação é selecionar os dados mais relevantes à previsão. Exemplos de pré-processamento são o emprego de Mapas de Kohonen, um tipo de rede neural artificial (RNA), mineração de dados e seleção aditiva (Bunnoon et al., 2010) e (Silva and Nascimento, 2017).

Os autores Al-Alawi and Islam (1996) fracionam os métodos de previsão de médio e longo prazo em três grandes grupos: métodos paramétricos, métodos de inteligência artificial (IA) e híbridos, que mesclam diferentes técnicas de IA e paramétricas com o intuito de aumentar a confiabilidade da previsão. Os métodos de inteligência artificial são, em geral, baseados na aplicação de RNA's, que possuem diversas arquiteturas e formas de treinamento, e nas máquinas de vetores de suporte, um conjunto de métodos empregados na classificação e regressão de dados.

A partir de um conjunto de exemplos $\left(x_{i}, y_{i}\right)$, em que os vetores $x_{i}$ representam as variáveis descritoras (atributos) e $y_{i}$ os valores da variável a ser descrita (rótulo), busca-se produzir um preditor, capaz de prever os valores futuros $y$ de uma série; esse processo de determinação é denominado treinamento.

Segundo Lorena and de Carvalho (2007) a máquina de vetores de suporte apresenta como pontos positivos principais a robustez frente à grandes volumes de dados, além da convexidade, que garante um mínimo global único. A possibilidade do emprego de funções de produto interno, em casos não lineares, também é destacada, já que podese construir hiperplanos simplificados em espaços de alta dimensão. As limitações da técnica residem na grande sensibilidade do modelo frente à escolha dos hiperparâmetros envolvidos.

Na Figura 1 é apresentado um fluxograma do processo de previsão de demanda. Nele são sintetizadas as etapas que podem estar envolvidas no processo de previsão.

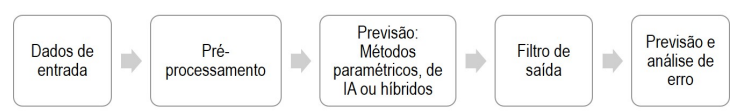

Figura 1. Fluxograma das etapas de previsão de demanda.

\section{PROPÓSITO}

A importância da predição da demanda energética reside, principalmente, no fato da mesma ser um produto, ou seja, há um viés econômico envolvido. Conhecer suas características, em diferentes horizontes temporais, influi, diretamente, no planejamento estratégico dos consumidores. Construir um modelo robusto e confiável, a partir de dados históricos, resiliente às possíveis modificações no comportamento de consumo de um cliente, conferelhe maior autonomia na decisão relacionada à compra de energia elétrica e firmamento de contratos relacionados. Além disso, a partir do conhecimento da demanda futura pode-se realizar o planejamento antecipado da construção de pequenas plantas de geração de energia limpa, em vias de suprir um possível consumo adicional e agir em consonância com as questões ambientais.
Dentro deste contexto, o propósito deste trabalho é aplicar um método de aprendizado supervisionado, conhecido como máquina de vetores de suporte voltada à regressão (SVR), para construir modelos de previsão de demanda de energia elétrica, a partir de um conjunto de dados históricos, como os de demanda da Universidade em estudo, e dados climáticos. Serão aplicados dois algoritmos diferentes na determinação dos hiperparâmetros: busca em grade e algoritmo genético. Por fim, os dois modelos gerados serão comparados por meio da métrica erro médio percentual absoluto (MAPE), selecionando-se o de melhor ajustamento e comparando o seu desempenho ao dos modelos desenvolvidos na literatura como em Prado (2011), Gonzalez et al. (2006) e Silva and Nascimento (2017), dentre outros.

\section{MÉTODOS}

Nesta seção serão descritos os métodos que foram empregados na solução do problema deste trabalho, contendo os dados históricos candidatos, que compreendem históricos de consumo e variáveis de viés climático, as etapas de préprocessamento e os métodos de determinação dos hiperparâmetros, além da formulação do algoritmo de aprendizado de máquina. Na Figura 2 é mostrado um fluxograma que representa o processo realizado.

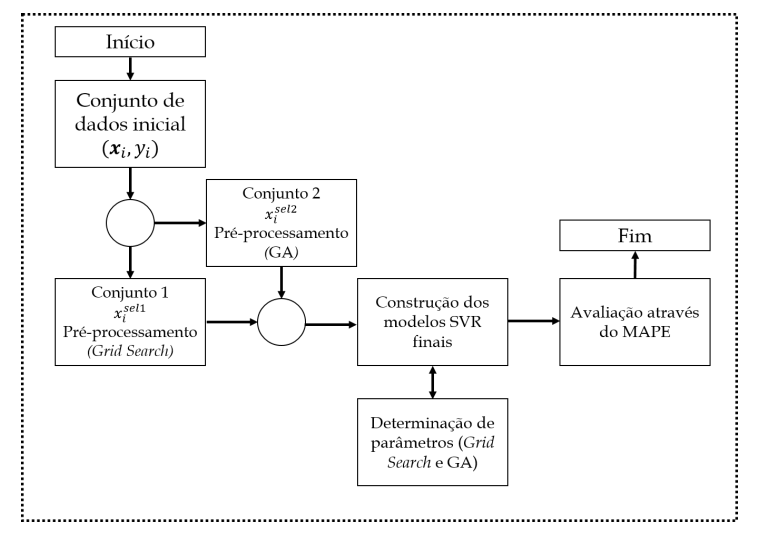

Figura 2. Fluxograma proposto do trabalho.

Na literatura, a avaliação das previsões são realizadas, quase que unanimemente, por meio do MAPE, o erro percentual absoluto médio, apresentado em (1), em que $y_{i}$ é o valor real dos dados, $\widehat{y}_{i}$ são os valores calculados por meio do modelo de previsão e $n$ o número de dados que compõem a previsão. Um MAPE de $1 \%$ aponta que há, em média, erros em $1 \%$ das $n$ previsões concretizadas pelo modelo (Bunnoon et al., 2010).

$$
M A P E=\frac{100}{n} \sum_{i=1}^{n}\left|\frac{\left(y_{i}-\widehat{y}_{i}\right)}{y_{i}}\right|
$$

\subsection{Preparação do conjunto de dados}

O conjunto de dados inicial $x_{i}$ foi composto inicialmente por elementos passíveis de avaliação de significância antes de sua integração ao modelo final buscado. De modo a avaliar a contribuição de um rol amplo de grandezas climáticas, foram incluídas no conjunto as seguintes: $x_{1}$ : 
Temperatura média mensal compensada; $x_{2}$ : Temperatura mínima mensal média; $x_{3}$ : Temperatura máxima mensal média; $x_{4}$ : Nível pluviométrico mensal; $x_{5}$ : Umidade. As séries históricas de cada variável supramencionada foram obtidas do portal virtual do INMET (INMET, 2018), o Instituto Nacional de Meteorologia, selecionando a estação Caxias do Sul.

Como apontado em Chen et al. (2004) e Dias (2007), a inclusão de valores defasados $n$-períodos de $y_{i}$ agregam ao modelo, logo serão incluídas duas defasagens ao conjunto $x_{i}$, sendo denominadas $x_{6}$ e $x_{7}$. O indicador de mês (1 a 12) figura como última variável, identificada por $x_{8}$. Compondo o conjunto $y_{i}$, estão disponíveis os dados de consumo mensal, referentes ao período compreendido entre 2013 e 2016, do campus sede da Universidade de Caxias do Sul.

\subsection{Pré-processamento}

A significância dos dados do modelo final foi apurado por meio da seleção aditiva (Forward Selection), como mostrado em Silva and Nascimento (2017). A partir de um conjunto de variáveis selecionadas vazio $\mathbf{x}_{i}^{\text {sel }}$, criam-se modelos individuais, para cada uma das variáveis contidas em $\mathbf{x}_{i}$, utilizando como métrica de avaliação o coeficiente de determinação $R^{2}$, mostrado em (2). A variável atrelada ao modelo de melhor desempenho é adicionada ao conjunto $\mathbf{x}_{i}^{\text {sel }}$. Após, repete-se o processo, para a próxima variável não selecionada do conjunto $\mathbf{x}_{i}$, e, a partir da construção de um modelo que a inclua, avalia-se se houve incremento em $R^{2}$; se sim, essa variável é adicionada ao conjunto $\mathbf{x}_{i}^{\text {sel }}$, se não, repete-se o processo para a próxima variável não selecionada, abrangendo todo o conjunto $\mathbf{x}_{i}$. O conjunto gerado ao fim contém as variáveis mais significativas para o modelo final.

$$
R^{2}=1-\left(\frac{\sum_{i=1}^{m}\left(y_{i}-\widehat{y}_{i}\right)^{2}}{\sum_{i=1}^{m}\left(y_{i}-\bar{y}_{i}\right)^{2}}\right)
$$

\subsection{Modelo SVR e Hiperparâmetros}

A partir das variáveis selecionadas na etapa de préprocessamento, contidas nos conjuntos $\mathbf{x}_{i}^{\text {sel1 }}$ (variáveis de entrada selecionadas para o algoritmo genético) e $\mathbf{x}_{i}^{\text {sel2 }}$ (variáveis de entrada selecionadas para a busca em grade), foram construídos os modelos de regressão finais. O objetivo do método SVR, a partir dos dados de entrada, é determinar um hiperplano $y(\mathbf{x})$ que, em sua formulação, apresente os menores desvios possíveis, compreendidos na faixa $[-\epsilon ; \epsilon]$ em relação aos valores reais $y_{i}$, ou seja, desvios que se encontrem dentro dessa margem são aceitos. Na Figura 3 é mostrado um conjunto de dados. Observa-se que há uma linha vermelha, que é a representação do hiperplano $(\mathbf{w} \cdot \mathbf{x})+\mathbf{b}$ que ajustará os dados. Ao seu redor há dois hiperplanos paralelos, a uma distância $\epsilon$. Esses hiperplanos são denominados margens, sendo que $\xi$ são os desvios dos dados que se encontram fora da região sombreada, compreendida entre as margens, denominada $\epsilon$-tubo insensível.

Conforme mostrado em Smola and Schölkopf (2004), a modelagem do SVR recai em um problema de minimização. Respeitando as premissas do modelo, deve-se definir um

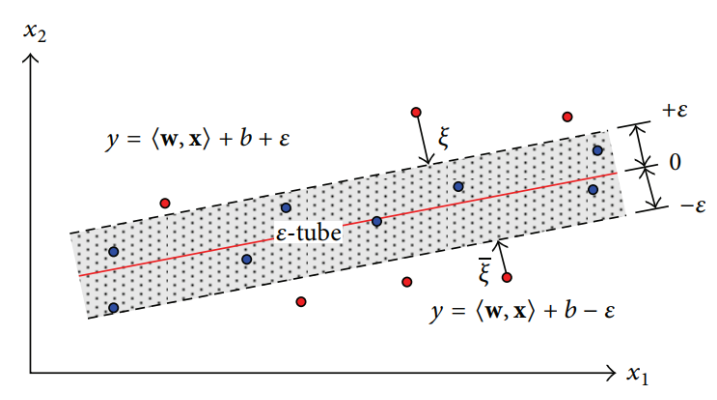

Figura 3. Cenário da SVR Yang et al. (2013).

hiperplano $y(\mathbf{x})$, que minimize $\|\mathbf{w}\|$ ou $\frac{1}{2}\|\mathbf{w}\|^{2}$, já que a distância do hiperplano à origem é $\frac{b}{\|\mathbf{w}\|}$. Deseja-se que a diferença entre os rótulos $y_{i}$ e o hiperplano determinado não seja superior a $\epsilon$. Aplicando-se multiplicadores de lagrange na resolução de tal problema não linear tem-se o mostrado em (3), que é a solução generalizada em função dos vetores de suporte. $K$ representa a função de núcleo, que realiza os produtos internos dos vetores em um espaço de alta dimensão.

$$
f(\mathbf{x})=\sum_{i=1}^{n}\left(\alpha_{i}-\alpha_{i}^{*}\right) K\left(\mathbf{x}_{\mathbf{i}} \cdot \mathbf{x}\right)+b
$$

Segundo Chen et al. (2004), para que o modelo de SVR implementado apresente um bom desempenho, é necessário determinar alguns parâmetros, denominados hiperparâmetros. São eles:

- $C$ - Penalização dos erros;

- Largura do $\epsilon$-tubo insensível;

- Núcleo de produto interno $K$ e seus parâmetros;

- Quantidade de dados históricos a serem considerados.

Na primeira abordagem, denominada Grid Search, ou busca em grade, faz-se uma procura exaustiva em um intervalo pré-determinado $(\gamma)$, em busca dos valores ótimos para o grupo de hiperparâmetros a serem determinados. De maneira geral, define-se um valor inicial, avalia-se o modelo por meio da validação cruzada. A técnica de validação cruzada é empregada para avaliação da capacidade de generalização de modelos de previsão. Nela, o conjunto de dados de treinamento disponível $\left(\mathbf{x}_{i} ; \mathbf{y}_{i}\right)$ é dividido em $k$ subgrupos, sendo $k-1$ subgrupos utilizados no treinamento do modelo e apenas um na validação, onde se analisará a qualidade do modelo. Esse procedimento é repetido $k$ vezes, de maneira circular, ou seja, todos os subgrupos farão o papel de validadores uma vez e assim repete-se o processo sucessivamente, percorrendo todo o intervalo definido. A grande restrição destes métodos é o alto custo computacional (Ito and Nakano, 2003).

A segunda abordagem na determinação dos hiperparâmetros do SVR dá-se pela aplicação do algoritmo genético (GA). Esse baseia-se na seleção natural. Inicialmente codifica-se o cromossomo, que, no que tange a este trabalho, está composto pelos parâmetros $C, \epsilon$ e $\sigma^{2}$, considerando-se um núcleo gaussiano. Segundo Smola et al. (1998), em geral, o núcleo gaussiano apresenta uma melhor performance sob condições gerais de suavidade e sua utilização deve ser considerada como tentativa inicial, quando não há informações específicas sobre o conjunto 
de dados disponível. Define-se uma função de aptidão; aqui será empregada novamente a validação cruzada. Inicialmente uma população de cromossomos, definidos de maneira aleatória, é criada. Logo, avalia-se os cromossomos gerados por meio da validação cruzada. Procede-se à solução pelo método da roleta, na qual os cromossomos são classificados de maneira proporcional ao resultado da avaliação realizada; nesta etapa deve-se escolher os mais preparados, sem desconsiderar os menos preparados, para manter a diversidade (Chen and Wang, 2007).

Os pares de cromossomos selecionados são recombinados, para que as melhores características dos geradores sejam preservadas. A introdução da mutação garante que a busca não fique condicionada somente à população inicial. Avalia-se a nova população; se essa apresentar melhores resultados que a anterior, há a substituição.

Como há dois conjuntos, empregando distintas técnicas de determinação de hiperparâmetros, será possível definir qual implica em um melhor desempenho, tanto sob a métrica de erro, quanto pelo número de avaliações da função, ou seja, pela ótica do custo computacional. As avaliações foram realizadas pelo cálculo do MAPE.

Os modelos foram implementados em MATLAB (MATLAB, 2015), com a utilização da biblioteca LIBSVM. Detalhes da implementação desta biblioteca são encontrados em Chang and Lin (2011) . Neste trabalho, o módulo $\epsilon$-support vector regression foi empregado.

\section{RESULTADOS}

Neste capítulo serão apresentados os resultados dos modelos apresentados na Seção 3. Inicialmente, serão expostos os resultados empregando a busca em grade e posteriormente os resultados por meio do emprego do algoritmo genético.

\subsection{Busca em grade}

Nas previsões para 12 meses compuseram o grupo de treinamento os dados referentes ao período compreendido entre o ano de 2012 e 2016. O grupo de validação foi composto pelos dados referentes ao ano de 2017. Empregou-se o intervalo de busca $2^{-25}$ a $2^{7}$ para $\gamma, 2^{-15}$ a $2^{0}$ para $\epsilon$ e $2^{-2}$ a $2^{10}$ para $C$. Por meio da variação do passo de busca $p$ buscou-se verificar a relação do MAPE e o número de avaliações da função. $\mathrm{O}$ modelo 1 possui passo de busca igual à 4 , o modelo 2 passo igual à 2 e o modelo 3 passo igual à 1,5. Na Tabela 1 é mostrado o panorama das avaliações realizadas, indicando as variáveis escolhidas, os erros e número de avaliações da função de treinamento.

Percebe-se que não há melhorias significativas no que tange a métrica de erro MAPE quando o intervalo de busca é extendido, por meio do descréscimo do passo, porém o número de avaliações é incrementado.

Na Figura 4 é mostrada a comparação entre os valores reais e os previstos pelo modelo de 12 meses, para o passo igual à 2 .

Na Tabela 2 é mostrada a evolução do algoritmo de seleção aditiva para todos cenários avaliados, destacando o erro MAPE e o coeficiente de determinação $R^{2}$, além das variáveis incluídas em cada etapa.

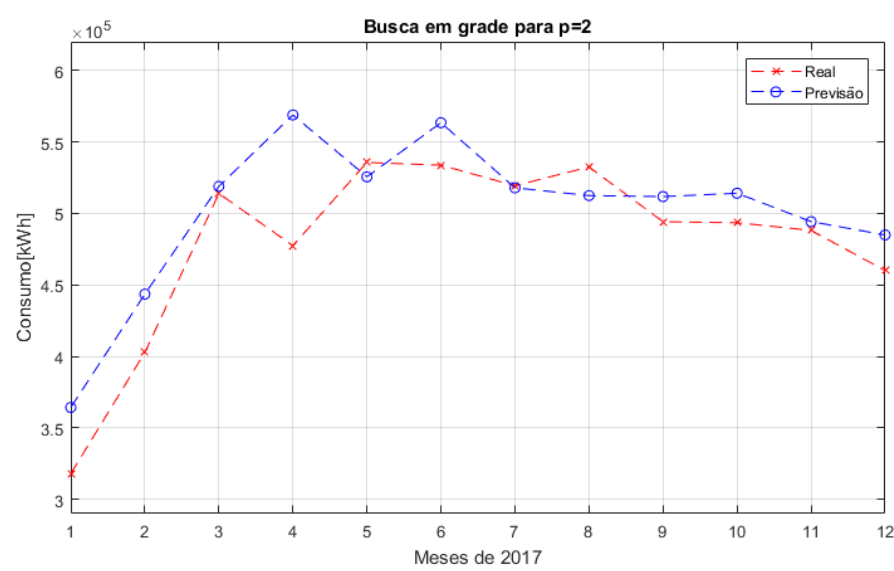

Figura 4. Previsão 12 meses com $p=2$.

Como já explanado, no método por seleção aditiva, as variáveis são avaliadas e as que efetivamente contribuem para o incremento da acurácia do modelo, por meio da análise do MAPE, são incluídas.

No modelo 1 foi obtido um $R^{2}$ de 0,674 ; no modelo 2 o valor do coeficiente foi 0,782 e no modelo 3 foi obtido 0,875 . Percebe-se que há o incremento do valor de $R^{2}$ ao reduzirse o passo, ou seja, melhora-se o ajustamento da curva, porém o MAPE não é reduzido à proporção. A partir disso conclui-se que um modelo mais ajustado aos dados, ou seja, que apresenta um erro médio quadrático menor (MSE) e um $R^{2}$ maior, não implica necessariamente em um modelo com maior assertividade nas previsões.

A variável $\mathbf{x}_{6}$, referente à valores de consumo defasados em um mês, integrou todos os modelos desenvolvidos, confirmando a relevância apontada em Chen et al. (2004) e Dias (2007). As variáveis $\mathbf{x}_{1}$ a $\mathbf{x}_{5}$, referentes à dados climáticos, como umidade e temperatura, em especial as mínimas médias mensais, também compuseram a grande parte dos modelos, comprovando a contribuição de tais grandezas na explicação do modelo. Indicadores de data (mês do ano) compuseram a grande maioria dos modelos, como em Silva and Nascimento (2017). Dados pluviométricos $\left(\mathbf{x}_{4}\right)$ e temperaturas máximas médias mensais $\left(\mathbf{x}_{3}\right)$ não representaram contribuição significativa ao modelo.

Nas previsões para 24 meses foram criados e avaliados modelos de previsão para um horizonte de 24 meses. O conjunto de treinamento do modelo foi composto pelos dados referentes ao período compreendido entre os anos de 2012 e 2015 e o conjunto de validação pelos dados referentes aos anos de 2016 e 2017. Foram empregados os mesmos intervalos de busca da subseção anterior e os três passos $p=4,2$ e 1,5. Na Figura 5 é mostrada a previsão para o passo $p=4$, que obteve o maior nível de acurácia sob a métrica do MAPE, apresentando um valor de 7,33\%, muito próximo ao das previsões de 12 meses. Na Tabela 3 são apresentados os valores obtidos de $R^{2}$ e MAPE em cada passo da seleção aditiva e as variáveis escolhidas.

As conclusões levantadas nas previsões de 12 meses também são válidas aqui. Ao incrementar-se o passo, o nível de ajustamento do modelo $R^{2}$ foi incrementado, o que não representou a diminuição do nível de assertividade, medido pelo MAPE. 
Tabela 1. Variação do passo na previsão para 12 meses.

\begin{tabular}{|c|c|c|c|c|}
\hline Modelo & Passo & MAPE & Variáveis escolhidas & Avaliações \\
\hline 1 & 4 & $6,24 \%$ & $\mathbf{x}_{6} \mathbf{x}_{8} \mathbf{x}_{7} \mathbf{x}_{1} \mathbf{x}_{5}$ & 4320 \\
\hline 2 & 2 & $5,89 \%$ & $\mathbf{x}_{6} \mathbf{x}_{8} \mathbf{x}_{2}$ & 24752 \\
\hline 3 & 1.5 & $6,48 \%$ & $\mathbf{x}_{6} \mathbf{x}_{8} \mathbf{x}_{2} \mathbf{x}_{5}$ & 65340 \\
\hline
\end{tabular}

Tabela 2. Iterações da seleção aditiva para 12 meses.

\begin{tabular}{|c|c|c|c|c|}
\hline Modelo & Etapa & Variáveis escolhidas & $R^{2}$ & MAPE \\
\hline \multirow{4}{*}{1} & 1 & $\mathbf{x}_{6}$ & 0,202 & $13,50 \%$ \\
\cline { 2 - 5 } & 2 & $\mathbf{x}_{6} \mathbf{x}_{8}$ & 0,553 & $10,80 \%$ \\
\cline { 2 - 5 } & 3 & $\mathbf{x}_{6} \mathbf{x}_{8} \mathbf{x}_{7}$ & 0,607 & $8,22 \%$ \\
\cline { 2 - 5 } & 4 & $\mathbf{x}_{6} \mathbf{x}_{8} \mathbf{x}_{7} \mathbf{x}_{1}$ & 0,755 & $6,91 \%$ \\
\cline { 2 - 5 } & 5 & $\mathbf{x}_{6} \mathbf{x}_{8} \mathbf{x}_{7} \mathbf{x}_{1} \mathbf{x}_{5}$ & 0,674 & $6,24 \%$ \\
\hline \multirow{4}{*}{3} & 1 & $\mathbf{x}_{6}$ & 0,199 & $14,17 \%$ \\
\cline { 2 - 5 } & 2 & $\mathbf{x}_{6} \mathbf{x}_{8}$ & 0,401 & $9,09 \%$ \\
\cline { 2 - 5 } & 3 & $\mathbf{x}_{6} \mathbf{x}_{8} \mathbf{x}_{2}$ & 0,782 & $5,89 \%$ \\
\cline { 2 - 5 } & 2 & $\mathbf{x}_{6}$ & 0,203 & $12,97 \%$ \\
\cline { 2 - 5 } & 3 & $\mathbf{x}_{6} \mathbf{x}_{8}$ & 0,596 & $8,56 \%$ \\
\cline { 2 - 5 } & 4 & $\mathbf{x}_{6} \mathbf{x}_{8} \mathbf{x}_{2} \mathbf{x}_{5}$ & 0,656 & $6,71 \%$ \\
\hline
\end{tabular}

Tabela 3. Iterações da seleção aditiva para 24 meses.

\begin{tabular}{|c|c|c|c|c|}
\hline Modelo & Etapa & Variáveis escolhidas & $R^{2}$ & MAPE \\
\hline \multirow{3}{*}{1} & 1 & $\mathbf{x}_{6}$ & 0,089 & $14,59 \%$ \\
\cline { 2 - 5 } & 2 & $\mathbf{x}_{6} \mathbf{x}_{1}$ & 0,465 & $11,89 \%$ \\
\cline { 2 - 5 } & 3 & $\mathbf{x}_{6} \mathbf{x}_{1} \mathbf{x}_{7}$ & 0,503 & $10,20 \%$ \\
\cline { 2 - 5 } & 4 & $\mathbf{x}_{6} \mathbf{x}_{1} \mathbf{x}_{7} \mathbf{x}_{8}$ & 0,586 & $8,28 \%$ \\
\cline { 2 - 5 } & 5 & $\mathbf{x}_{6} \mathbf{x}_{1} \mathbf{x}_{7} \mathbf{x}_{8} \mathbf{x}_{3}$ & 0,590 & $7,33 \%$ \\
\hline \multirow{3}{*}{3} & 1 & $\mathbf{x}_{6}$ & 0,103 & $15,97 \%$ \\
\cline { 2 - 5 } & 2 & $\mathbf{x}_{6} \mathbf{x}_{2}$ & 0,592 & $11,79 \%$ \\
\cline { 2 - 5 } & 1 & $\mathbf{x}_{6}$ & 0,103 & $15,97 \%$ \\
\cline { 2 - 5 } & 3 & $\mathbf{x}_{6} \mathbf{x}_{2}$ & 0,570 & $11,79 \%$ \\
\cline { 2 - 5 } & 4 & $\mathbf{x}_{6} \mathbf{x}_{2} \mathbf{x}_{8} \mathbf{x}_{8} \mathbf{x}_{5}$ & 0,564 & $10,43 \%$ \\
\cline { 2 - 5 } & 5 & $\mathbf{x}_{6} \mathbf{x}_{2} \mathbf{x}_{8} \mathbf{x}_{5} \mathbf{x}_{7}$ & 0,7606 & $9,29 \%$ \\
\hline
\end{tabular}

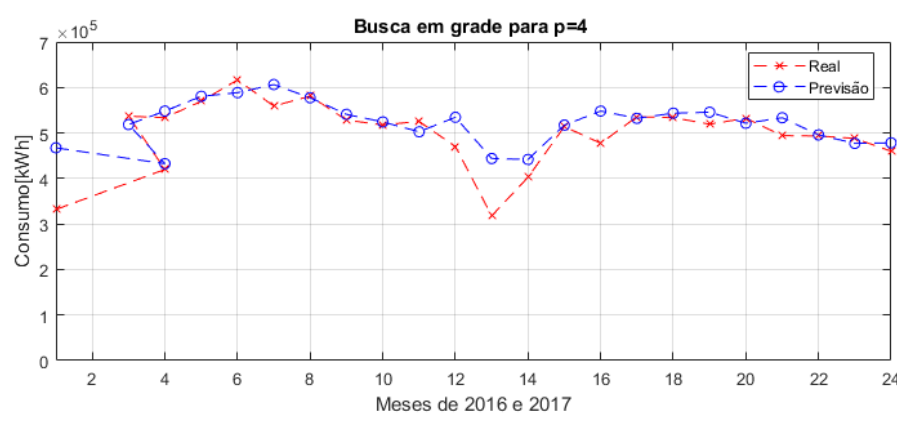

Figura 5. Previsão 24 meses com $p=4$.

\subsection{Algoritmo Genético}

Nesta seção serão apresentados os modelos criados com a utilização do algoritmo genético na seleção dos hiperparâmetros. Inicialmente, para avaliar o impacto das mudanças dos parâmetros no desempenho dos modelos foram realizadas previsões para 12 meses à frente e comparado o MAPE. $\mathrm{O}$ conjunto de treinamento foi composto pelos dados referentes aos anos de 2012 a 2016 e o conjunto de validação pelos dados do ano de 2017. Visto que o algoritmo genético é um método estocástico, mesmo sob o mesmo conjunto de parâmetros, resultados diferentes podem ser obtidos. Para isso foram realizadas cinco execuções e a média aritmética foi tomada.

Mantendo-se a probabilidade de cruzamento em 0,8 , a de mutação em 0,05 e o número de gerações em 100, ou seja, os valores sugeridos em Chen et al. (2004), o tamanho da população foi variado de 8 a 200 indivíduos, em 6 passos. A população inicial foi alimentada por uma matriz cujos elementos encontravam-se no intervalo entre $2^{-20}$ e $2^{20}$, espaçados igualmente. O número de avaliações da função foi contabilizado, assim como as variáveis escolhidas na seleção aditiva, o MAPE e o $R^{2}$ de cada modelo. Estes dados são mostrados na Tabela 4.

Percebe-se que o aumento no tamanho da população, a partir de 48 indivíduos, não produz uma redução significativa do MAPE, que oscila em uma faixa entre 5,72\% e $8,35 \%$, porém incrementa substancialmente o número de avaliações da função de treinamento. Uma população muito pequena, como 8, limita o espaço de busca e não garante a diversidade, logo os resultados são inferiores aos apresentados em populações maiores. Com base nestas avaliações foi concluído que o tamanho 8 proposto por Chen et al. (2004) não trouxe os melhores resultados aos modelos deste trabalho. 
Tabela 4. Variação do tamanho da população

\begin{tabular}{|c|c|c|c|c|c|}
\hline Modelo & Tamanho População & Variáveis & $R^{2}$ & MAPE & Avaliações \\
\hline 1 & 8 & $\mathbf{x}_{6}$ e $\mathbf{x}_{7}$ & 0,312 & $10,06 \%$ & 16256 \\
\hline 2 & 48 & $\mathbf{x}_{6} \mathbf{x}_{7} \mathbf{x}_{8}$ e $\mathbf{x}_{1}$ & 0,714 & $7,51 \%$ & 106368 \\
\hline 3 & 86 & $\mathbf{x}_{6} \times \mathbf{x}_{8}$ & 0,644 & $8,35 \%$ & 196510 \\
\hline 4 & 124 & $\mathbf{x}_{6} \mathbf{x}_{2} \quad \mathbf{x}_{8}$ & 0,736 & $5,72 \%$ & 250356 \\
\hline 5 & 162 & $\mathbf{x}_{6} \mathbf{x}_{2} \mathbf{x}_{1} \mathrm{e} \mathbf{x}_{8}$ & 0,746 & $6,02 \%$ & 301932 \\
\hline 6 & 200 & $\mathbf{x}_{6} \mathbf{x}_{2} \mathbf{x}_{1} \mathrm{e} \mathbf{x}_{8}$ & 0,770 & $7,03 \%$ & 459600 \\
\hline
\end{tabular}

Empregando os parâmetros que conduziram aos melhores resultados nas avaliações realizadas, os modelos finais de previsão para 12 e 24 meses foram construídos. São eles:

- Número de gerações: 100

- Tamanho da população: 124

- Probabilidade de cruzamento: 0,8

Na Figura 6 é mostrada a previsão para 12 meses, cujas variáveis selecionadas foram $\mathbf{x}_{6}, \mathbf{x}_{8}$ e $\mathbf{x}_{2}$, e na Figura 6 para 24 meses, cujas variáveis escolhidas foram $\mathbf{x}_{6}, \mathbf{x}_{8}$ e $\mathbf{x}_{1}$. O MAPE para a primeira foi de $5,72 \%$ e para a segunda de $9,05 \%$.

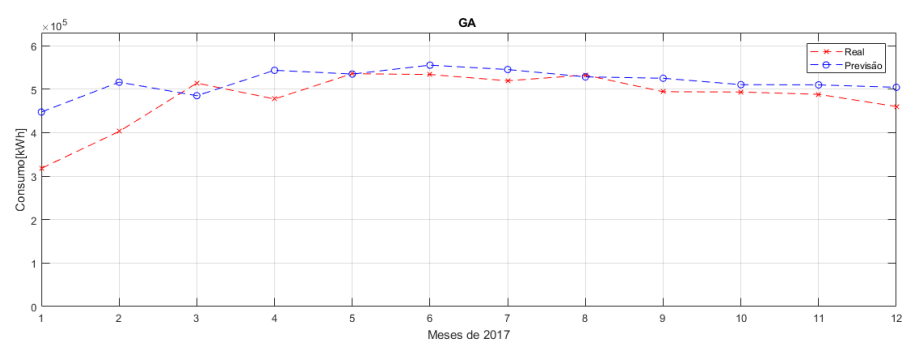

Figura 6. Previsão para 12 meses empregando GA.

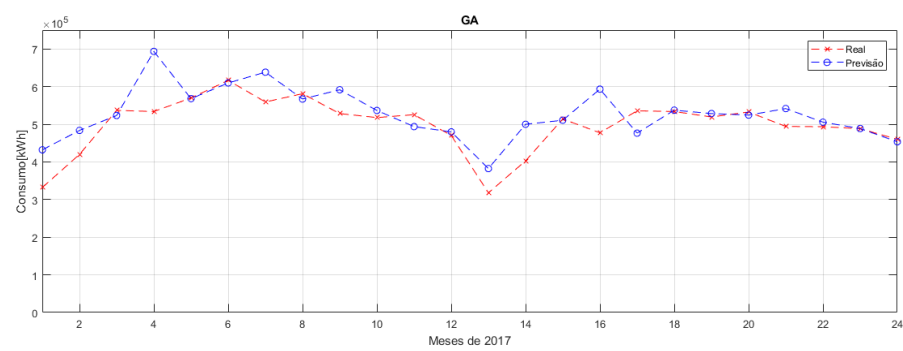

Figura 7. Previsão para 24 meses empregando GA.

Realizando-se uma comparação dos modelos desenvolvidos neste trabalho, cujos desempenhos foram os melhores em cada cenário analisado, tem-se o mostrado na Tabela 5.

\section{DISCUSSÕES}

Para promover uma comparação justa à literatura esta dar-se-á por meio do MAPE, já que esta métrica de erro é quase que absoluta na avaliação de modelos de previsão. O modelo de maior assertividade desenvolvido neste trabalho obteve um MAPE de $5,72 \%$, onde foi empregado o algoritmo genético na determinação dos hiperparâmetros, em um horizonte de 12 meses. O modelo de 24 meses de melhor performance obteve MAPE de $7,33 \%$, onde a busca em grade foi empregada. Na Tabela 6 são apresentados os valores de MAPE dos modelos encontrados na literatura
O melhor modelo deste trabalho, cujo MAPE foi de 5,72\%, distanciou-se em 4,26\% do melhor modelo da literatura, de Torkzadeh et al. (2014). Há de pontuar-se que o autor empregou um rol de variáveis mais amplo, incluindo informações econômicas, além de um período maior de dados históricos que o empregado neste trabalho.

\section{CONCLUSÕES}

Neste trabalho foram implementados modelos de previsão de demanda utilizando o método de aprendizagem supervisionado conhecido como máquina de vetores de suportes. Para o ajuste dos hiperparâmetros do modelo foi proposta a comparação de dois métodos: a busca em grade e o algoritmos genético, ambos largamente empregados na literatura. A busca em grade, ao promover ajustes nos modelos, pode implicar em um custo computacional significativo, ao incrementar o espaço de busca, enquanto o método evolutivo do algoritmo genético pode fornecer soluções de superior qualidade, porém exige um número elevado de avaliações da função, quando escolhidas grandes populações de indivíduos, e/ou a avaliação de um grande número de gerações. Além disso, enquanto não é necessário possuir conhecimentos prévios do espaço de busca no emprego do algoritmo genético, já que é evolutivo e converge aos hiperparâmetros ótimos, na busca em grade o espaço de busca pode ficar limitado, podendo-se não atingir resultados adequados. A partir da aplicação da técnica de seleção aditiva foi possível conceber modelos com um número enxuto de variáveis explicativas, confirmando a qualidade de tal técnica na seleção de variáveis quando um grande número delas está presente. Em trabalhos futuros podem ser incluídas no rol de variáveis explicativas, a serem analisadas, as referentes ao número de matrículas ativas na universidade, já que isso impacta no volume de dependências utilizadas na Universidade, e consequentemente no consumo de energia elétrica. Também seria possível aplicar outros tipos de técnicas, como redes neurais artificiais e métodos Box-Jenkins, além de métodos distintos na determinação de hiperparâmetros, a fim de comparar se são mais eficientes que os abordados neste trabalho.

\section{REFERÊNCIAS}

Al-Alawi, S.M. and Islam, S.M. (1996). Principles of electricity demand forecasting. i. methodologies. Power Engineering Journal, 10(3), 139-143.

Bunnoon, P., Chalermyanont, K., and Limsakul, C. (2010). A computing model of artificial intelligent approaches to mid-term load forecasting: a state-of-the-art-survey for the researcher. International Journal of Engineering and Technology, 2(1), 94 . 
Tabela 5. Comparação entre modelos.

\begin{tabular}{|c|c|c|c|}
\hline Horizonte & GA/GS & Variáveis candidatas & MAPE \\
\hline 12 meses & GA & Todas & $5,72 \%$ \\
\hline 12 meses & GS & Todas & $5,89 \%$ \\
\hline 24 meses & GA & Todas & $9,05 \%$ \\
\hline 24 meses & GS & Todas & $7,33 \%$ \\
\hline
\end{tabular}

Tabela 6. Comparação com a literatura.

\begin{tabular}{|c|c|c|c|}
\hline Autor & Método & Horizonte & MAPE \\
\hline PRADO (2011) & SARIMA & 12 meses & $7,5 \%$ a $15,89 \%$ \\
\hline $\begin{array}{c}\text { GIAROLA; TAKENAKA; } \\
\text { ROCHA (2014) }\end{array}$ & SARIMA & 17 meses & $7,50 \%$ \\
\hline GONZALEZ et al. (2006) & RNA e ARIMA & 60 meses & $1,89 \%$ a $3,80 \%$ \\
\hline PAN; LEE (2012) & RNA e SVR & 1 mês & $3,51 \%$ a $9,64 \%$ \\
\hline BUNNON (2012) & RNA & 34 meses & $4,70 \%$ a $6,51 \%$ \\
\hline SILVA (2017) & RLM & 12 meses & $<5 \%$ \\
\hline TORKZADEH et al. (2014) & RLM & 24 meses & $1,46 \%$ a $3,68 \%$ \\
\hline CHEN; CHANG et al. (2014) & SVR & 1 mês & $1,95 \%$ \\
\hline Modelo 12 meses & SVR+GA & 12 meses & $5,72 \%$ \\
\hline Modelo 24 meses & SVR+GS & 24 meses & $7,33 \%$ \\
\hline
\end{tabular}

Chang, C.C. and Lin, C.J. (2011). LIBSVM: A library for support vector machines. ACM Transactions on Intelligent Systems and Technology, 2, 27:1-27:27. Software available at http://www.csie.ntu.edu.tw/ cjlin/libsvm.

Chen, B.J., Chang, M.W., et al. (2004). Load forecasting using support vector machines: A study on eunite competition 2001. IEEE transactions on power systems, 19(4), 1821-1830.

Chen, K.Y. and Wang, C.H. (2007). Support vector regression with genetic algorithms in forecasting tourism demand. Tourism Management, 28(1), 215-226.

Dias, M.S. (2007). O uso de Máquina de Suporte Vetorial para Regressao (SVR) na Estimaçao da Estrutura a Termo da Taxa de Juros do Brasil. Ph.D. thesis, PUCRio.

Ferreira, R.V. (2006). Previsão de demanda: Um estudo de caso para o sistema interligado nacional. Universidade Federal de Minas Gerais-Escola de EngenhariaPrograma de Pós-Graduação em Engenharia ElétricaBelo Horizonte-2006.

Gonzalez, Romera, E., Jaramillo, Moran, M.A., and Carmona, Fernandez, D. (2006). Monthly electric energy demand forecasting based on trend extraction. IEEE Transactions on power systems, 21(4), 1946-1953.

INMET (2018). Estações e dados. $<$ http://www.inmet.gov.br/portal $>$. Acessado em: 24 mar. 2018.

Ito, K. and Nakano, R. (2003). Optimizing support vector regression hyperparameters based on cross-validation. In Neural Networks, 2003. Proceedings of the International Joint Conference on, volume 3, 2077-2082. IEEE.

Khuntia, S.R., Rueda, J.L., and van der Meijden, M.A. (2016). Forecasting the load of electrical power systems in mid-and long-term horizons: a review. IET Generation, Transmission \& Distribution, 10(16), 3971-3977.

Lorena, A.C. and de Carvalho, A.C. (2007). Uma introdução às support vector machines. Revista de Informática Teórica e Aplicada, 14(2), 43-67.
MATLAB (2015). Version R2015a. The MathWorks Inc., Natick, Massachusetts.

Prado, J.R.d. (2011). Modelos para demanda e consumo de energia elétrica utilizando séries temporais na universidade federal de lavras. Universidade Federal de Lavras.

Silva, A.C.d. and Nascimento, A.H.d. (2017). Previsão anual de demanda de energia elétrica. CBQEE, Curitiba.

Smola, A.J. and Schölkopf, B. (2004). A tutorial on support vector regression. Statistics and computing, 14(3), 199-222.

Smola, A.J., Schölkopf, B., and Müller, K.R. (1998). The connection between regularization operators and support vector kernels. Neural networks, 11(4), 637-649.

Torkzadeh, R., Mirzaei, A., Mirjalili, M.M., Anaraki, A.S., Sehhati, M.R., and Behdad, F. (2014). Medium term load forecasting in distribution systems based on multi linear regression \& principal component analysis: A novel approach. In Electrical Power Distribution Networks (EPDC), 2014 19th Conference on, 66-70. IEEE.

Yang, C.Y., Chang, L.C., Samani, H., and Nakatsu, R. (2013). Ameliorated austenite carbon content control in austempered ductile irons by support vector regression. In Mathematical Problems in Engineering, 2013, 9. doi: $10.1155 / 2013 / 362601$. 